\title{
An Integrated 4-element Slot-Based MIMO and an UWB Sensing Antenna System for CR Platforms
}

\author{
Rifaqat Hussain, Mohammad S. Sharawi and Atif Shamim
}

\begin{abstract}
This paper presents a novel integrated antenna system for cognitive radio (CR) applications. The design consists of a compact 4 element reconfigurable annular slot based multiple-input-multiple-output (MIMO) antenna system integrated within an ultra-wide-band (UWB) sensing antenna. All the antenna elements are planar in structure and designed on a single substrate $(R O-4350)$ with dimensions $60 \times 120 \times 1.5$ $\mathrm{mm}^{3}$. The frequency reconfigurable slot based MIMO antenna system is tuned over a wide frequency band from $1.77 \mathrm{GHz}$ to $2.51 \mathrm{GHz}$ while the UWB sensing antenna is covering from $0.75 \sim 7.65 \mathrm{GHz}$ The proposed antenna system is suitable for CR enabled wireless devices. The envelope correlation coefficient (ECC) did not exceed 0.248 in the entire operating band of the MIMO antenna part. The maximum measured gain of the MIMO antenna is $3.2 d B i$ with maximum efficiency of $81 \%$.
\end{abstract}

Keywords: cognitive radio, MIMO, reconfigurable antennas, UWB antenna

\section{INTRODUCTION}

In modern wireless communication systems, high data rate and efficient spectrum utilization are highly desirable as new features and services are continuously added to wireless devices and mobile terminals. High data rate requirements can be achieved by utilizing multiple-input-multiple-output (MIMO) systems with multi/wideband or covering several frequency bands in cognitive radio (CR) platforms. A CR based system senses the unoccupied or underutilized frequency bands, then switches the frequency band of operation to the free one, hence achieving better spectrum utilization [1]. A frequency reconfigurable MIMO antenna system is a key element in second generation CR based applications [2].

Several MIMO reconfigurable antenna designs were reported in literature for $\mathrm{CR}$ applications [3]-[6]. In [3], a 2-element printed inverted F-shape antenna (PIFA) was presented. The antenna covered frequency bands of $2.3 \sim 2.4 \mathrm{GHz}, 2.5 \sim 2.7 \mathrm{GHz}$, and $3.4 \sim 3.6 \mathrm{GHz}$ with single element dimensions of $10 \times 10 \times 6.4 \mathrm{~mm}^{3}$. Similarly, a MIMO PIFA was presented in [4]. The frequency bands covered were $2.1 \sim 2.9 \mathrm{GHz}$ with single element dimensions of $16 \times 33 \times 5 \mathrm{~mm}^{3}$. In [5], a frequency reconfigurable MIMO slot antenna was presented. The resonance frequency bands obtained were from $2.4 \sim 2.5 \mathrm{GHz}$, 4.9 5.725 GHz and 4.9 5.725 $\mathrm{GHz}$. The fabricated board dimensions were $46 \times 20 \times 1.6 \mathrm{~mm}^{3}$ with single antenna element size of $3 \times 5$ $\mathrm{mm}^{2}$. A combination of microstrip loop MIMO antenna system along with slot antennas were presented in [6]. The MIMO antenna covered $1.71 \sim 1.88 \mathrm{GHz}$ and $2.5 \sim 2.7 \mathrm{GHz}$ bands. The second slot antenna covered $0.496 \sim 0.862 \mathrm{GHz}$. The board used was of dimensions $150 \times 150 \mathrm{~mm}^{2}$.

CR antennas with both reconfigurable MIMO and sensing antennas were presented [7]-[10]. In [7], a frequency reconfigurable communication antenna was presented covering $2.1 \sim 3 \mathrm{GHz}, 3 \sim 3.4$, 3.4 5.56, 5.4 6.2 GHz and 6.3 10 GHz while the UWB antenna covered 2.1 10 GHz. Both the reconfigurable and UWB antennas

R. Hussain and M. S. Sharawi are with the Electrical Engineering Department, King Fahd University for Petroleum and Minerals (KFUPM) Box 34, Dhahran, 31261 Saudi Arabia, Email: \{rifaqat, msharawi\}@kfupm.edu.sa A. Shamim is with the Computer, Electrical and Mathematical Science and Engineering Division, King Abdullah University (KAUST) of Science and Technology, Thuwal 23955-6900, Saudi Arabia, Email:atif.shamim@kaust.edu.sa were of modified monopole type. The whole antenna design was realized on ground (GND) plane area of $50 \times 70 \mathrm{~mm}^{2}$. In [8], a two element frequency reconfigurable MIMO PIFA antenna system was presented along with a modified monopole based UWB sensing antenna on a board volume of $65 \times 120 \times 5.8 \mathrm{~mm}^{3}$. The reconfigurable communication antenna was covering several frequency bands in the frequency range from $0.7 \mathrm{GHz}$ to $3 \mathrm{GHz}$ while the sensing antenna covered a very wide range of frequency bands from 0.71 to $3.6 \mathrm{GHz}$. In [9], monopole based reconfigurable MIMO filtenna was presented for $\mathrm{CR}$ applications along with sensing antenna for interweave and underlay $\mathrm{CR}$ systems. Both the sensing and the reconfigurable antennas were designed for the frequency range $3 \sim 6$ $\mathrm{GHz}$ with board dimensions of $70 \times 80 \mathrm{~mm}^{2}$. In [10], a two element frequency reconfigurable PIFA based MIMO antenna was integrated with an UWB sensing antenna. A unique combination of PIN and varactor diodes were utilized to switch the frequency bands of operation and sweep the resonance frequencies over the tuned band. The reconfigurable MIMO antenna covered frequency bands: 1.1 $\mathrm{GHz}, 2.48 \mathrm{GHz}, 0.573 \sim 0.680 \mathrm{GHz}$ and $0.834 \sim 1.120 \mathrm{GHz}$ while the sensing antenna covered a band from $0.72 \sim 3.44 \mathrm{GHz}$. The substrate dimensions were $65 \times 120 \times 0.8 \mathrm{~mm}^{3}$. The antennas presented in [8][10] were 2-element MIMO frequency reconfigurable antenna designs along with an UWB sensing one. None of the presented designs had 4-element frequency agile MIMO antennas along with UWB sensing antenna for CR applications with small size.

The proposed antenna provides a wide sweep of resonance frequencies over a wide band using varactor diodes. Most of the open slot-based antenna designs are made reconfigurable using PIN diodes, operating at some distinct frequency bands. Open slot-based antennas are difficult to load reactively and hence it is quite challenging to get the resonance frequency sweeps over a wide-band. In addition, any shift in the resonance frequency between simulated and measured results can be easily compensated for in case of annular/closed loop slot-based antenna due to the smooth variation of resonance frequencies and hence it is a better option than the PIN diode based open slot-based antenna designs. The proposed annular slotbased antenna design is planar in structure and hence can be easily integrated with other circuit components. On the other hand, inverted F-shaped antennas are non-planar in structure and hence it is quite challenging to integrate them with other circuit components on the main PCB board. Hence, the proposed antenna design is a better option than open-slot based antennas or PIFA ones.

In this work, a 4-element annular slot-based MIMO antenna system integrated within an UWB sensing antenna is presented. The proposed MIMO antenna system is made reconfigurable using a single varactor diode per antenna element. The MIMO antenna system is tuned over wide and continuous frequency bands from $1.77 \mathrm{GHz}$ to $2.51 \mathrm{GHz}$. The MIMO antenna covers the well-known frequency standards of GSM1800 / LTE / UMTS / WLAN along with several others. A compact modified UWB monopole based sensing antenna is presented to cover from 0.75 to $7.65 \mathrm{GHz}$. The distinguishing feature of the proposed design is the way we integrated the 4-antenna elements within the UWB antenna on a planar structure within a compact size. The proposed CR antenna system was realized on substrate volume 
of $60 \times 120 \times 1.5 \mathrm{~mm}^{3}$.

The rest of the paper is organized as follows. Sections II describes the antenna system design. Simulation and measurement results for the UWB and MIMO antenna systems are given in Section III. The conclusions are provided in Section IV.

\section{Design Details of the CR Antenna System}

The geometry of the 4-element frequency agile slot based MIMO antenna system along with the UWB sensing antenna is shown in Fig. 1, while the fabricated model is shown in Fig. 2. The CR antenna system was designed on an $R O-4350$ substrate with a relative permittivity $\left(\epsilon_{r}\right)$ of 3.48 , loss tangent of 0.0036 and thickness $1.5 \mathrm{~mm}$. The antennas were realized on board dimensions of $60 \times 120 \mathrm{~mm}^{2}$ to check the smallest size we can use to accommodate the design. The proposed CR antenna system is for wireless handheld devices and mobile terminals with an objective to be accommodated on a typical smart phone back-plane size.

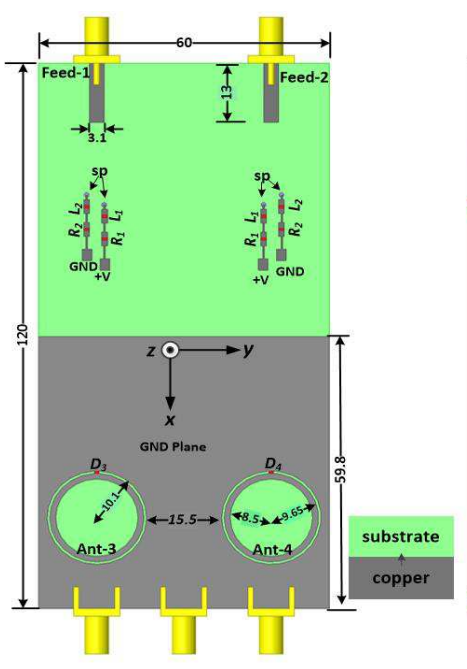

(a)

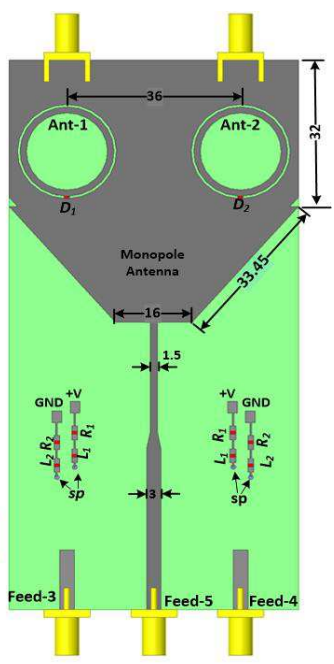

(b)
Figure 1: Proposed MIMO antennas system for CR platform (a) Top view (b) Bottom view - All dimensions are in millimeters (mm).

\section{A. Design Details of the UWB Sensing Antenna}

The CR antenna system consists of UWB sensing antenna and reconfigurable communication antenna system. Both antenna systems work sequentially. At first, the UWB antenna is utilized to sense the non-occupied or under-utilized frequency bands while in the second stage, the reconfigurable communication antenna switch its frequency band of operation to the new assigned band [11].

Fig. 1(b) shows the configuration of the modified monopole based wine-glass-shaped UWB sensing antenna. The proposed antenna design is a miniaturized version (via altering edge bends and reference plane size and separation) of the ones presented in [8], [10], but with improved performance. The proposed antenna design covered much wider bandwidth (BW) and better radiation characteristics. The antenna was fed by a $50 \Omega$ microstrip line with different step impedances for better impedance matching. The $45^{\circ}$ side of the antenna was optimized to resonate the antenna over a wide band. Two circular slots of radius $9.65 \mathrm{~mm}$ were etched out from the antenna surface. These slots helped in enhancing the impedance BW by 67 $\mathrm{MHz}$. Moreover, the same slots were also utilized as reconfigurable communication antennas for CR applications. Parametric sweeps were performed to optimize the various lengths of the UWB antenna including the length of the microstrip feeding line. The dual purpose GND plane for UWB antenna as shown Fig. 1(a) acted as a reference plane for the sensing antenna and also accommodated two annular slot-based reconfigurable MIMO antennas.

In the proposed design, the reference GND plane of the MIMO antenna system is intelligently converted to work as an UWB sensing antenna for the desired band of operation. The dimensions of the GND plane were optimized to cover an UWB frequency range. It is quite challenging and almost impossible to design a separate UWB monopole antenna to cover frequency bands below $2 \mathrm{GHz}$, with the size constraint of a PCB board of typical smart phone back-plane size. The proposed design is best so far in terms of accommodating and UWB sensing antenna along with 4-element frequency reconfigurable MIMO antenna system integrated within a compact size.

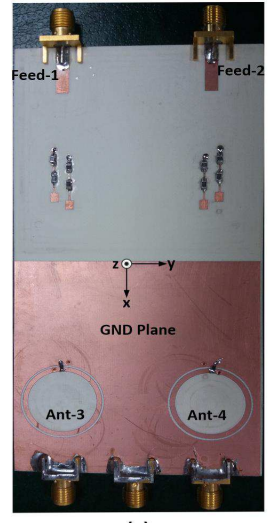

(a)

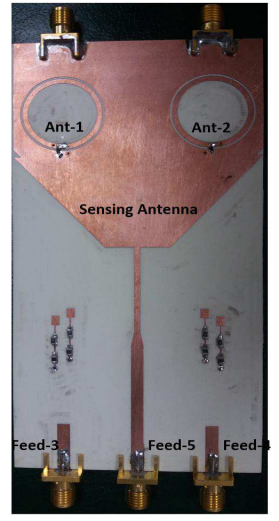

(b)
Figure 2: Fabricated prototype (a) Top view (b) Bottom view.

\section{B. Design Details of the MIMO Reconfigurable Antenna}

The geometry of the proposed frequency agile MIMO antenna system is shown in Fig. 1. The top and bottom layers of the board contained 2-element slot antennas on each side. The proposed design was basically a printed modified monopole based UWB antenna that was intelligently converted into an integrated CR antenna system without much affecting the UWB performance. The GND plane and monopole antenna structure were optimized to accommodate 2element slot-based reconfigurable antennas on each side, designated as Ant-1, Ant-2, Ant-3 and Ant-4, respectively, as shown in Fig. 1.

The design procedure of a single slot antenna was started with single annular slot antenna fed with $50 \Omega$ microstrip line. In the slot-based antenna design, the dimensions of the slot determine its resonance frequency. In the proposed antenna design, an electrical length of $\lambda / 2$, corresponds to $2.9 \mathrm{GHz}$ resonance band without the reactive loading. Varactor diodes were added on the annular slot edge to bring down the resonance frequency to lower bands. This in fact matches the input impedance of the antenna to $50 \Omega$ at the lower frequency band. The final optimized antenna dimensions were selected to cover the maximum number of wireless communication standards bands. The limiting factors for wide frequency tuning for the given MIMO antenna system are: the limited varactor diode capacitance below $1 p F$ range, the exact position of varactor diode to effectively load the antenna and the lack of modeling flexibility of varactor diodes in HFSS.

Each antenna element was optimized and parametric sweeps were performed for varactor diode placement on the specific location to reactively load the slot. The position of the varactor diode has maximal effect on the antenna resonance. Varactor diodes $\left(D_{1}, D_{2}\right.$, $D_{3}$ and $D_{4}$ ) connect the inner and outer parts of the annular slot with capacitive reactance loading. The varactor diode terminals were connected to a biasing circuit using two shorting pins (sp)/vias as shown Fig. 1. 
The varactor diode biasing circuitry consisted of a series combination of an RF choke of $1 \mu \mathrm{H}\left(L_{1}\right.$ and $\left.L_{2}\right)$ and $2.1 \mathrm{k} \Omega$ resistors $\left(R_{1}\right.$ and $\left.R_{2}\right)$ connected to the two terminals of the varactor diode. An RF choke was used to block the RF signal from entering the power supply while while the resistors were used as current limiter in the biasing circuitry. The RF choke used was MLF-2012 type with typical self-resonance frequency (SRF) value of $160 \mathrm{MHz}$. The varactor diode was serving to isolate the DC current from entering the radiating structure. The two RF chokes connected to the two terminals of the varactor diode, as well as the radiating structure, were utilized to block the RF signal from both ends of the antenna terminals to the power supply and hence avoid any radiation from the biasing circuitry. The current limiting resistors on both sides of the varactor diode were used to allow current to flow in the circuit within the threshold. The diodes were utilized to tune the resonance frequency over a wide operating band. The varactor diodes used were SMV 1233.

The proposed MIMO antenna design was fabricated using an LPKF S103 machine at KFUPM. Fig. 2(a) shows the top view of the fabricated design having 2-element reconfigurable slot antennas, feeding lines, GND plane and biasing circuitry for Ant-1 \& Ant2, while Fig. 2(b) shows the bottom layer containing the 2-element reconfigurable slot antenna and biasing circuit for Ant-3 \& Ant-4.

\section{Simulation and Measurement Results}

The complete integrated 4-element slot-based reconfigurable MIMO antenna system along with the UWB sensing antenna was modeled and simulated using $\operatorname{HFSS}^{T M}$. The antenna dimensions were optimized to cover most of the cellular wireless standards. The S- parameters were measured using an Agilent N9918A vector network analyzer. The gain patterns and efficiencies were measured at King Abdullah University of Scinece and Technology (KAUST), Saudi Arabia, using a SATIMO Starlab anechoic chamber.

\section{A. UWB Sensing Antenna}

The proposed UWB sensing antenna was based on a geometrically modified monopole printed structure with an UWB frequency coverage from 0.75 to $7.65 \mathrm{GHz}$. Most of the UWB antenna designs cited in literature were operating above $3 \mathrm{GHz}$ and very few were found in $2 \mathrm{GHz}$ operation band such as [12]-[14], keeping in mind the area constraint. The size of the proposed antenna system is compact and the structure is planar and simple with very good performance metrics. The present design also provides twice the covered bands compared to [8], [10] via careful geometrical optimization.

The simulated and measured reflection coefficients curves of the UWB antenna are given in Fig. 3(a). The proposed UWB antenna covered a frequency band from 0.75 to $7.65 \mathrm{GHz}$ (-6 $d B \mathrm{BW}$ ). A good agreement between simulated and measured results was obtained. The reconfigurable slot antenna elements were integrated within the monopole structure and hence it was critical to analyze the mutual coupling between them. Fig. 3(b) shows the isolation between the UWB band and reconfigurable MIMO antenna elements (between UWB antenna \& Ant-1 and UWB antenna \& Ant-3). Good isolation results were observed with a worst case isolation of $12 d B$ in the entire resonance band. The varactor diodes were supplied with $0 \mathrm{~V}$ while measuring $S_{55}$ and $S_{k 5}$. All the MIMO antenna elements were terminated with $50 \Omega$ load when we were measuring the $S_{55}$ curve and in the case of $S_{k 5}$, the $k^{t h}$ port of MIMO antenna element and input port of sensing antenna were active with zero biasing voltage to the corresponding varactor diode. The maximum coupling between sensing antenna and MIMO antenna were changing with different biasing conditions.

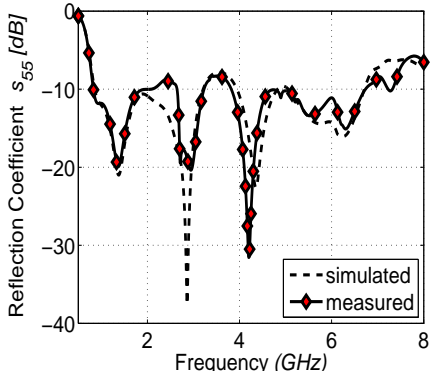

(a)

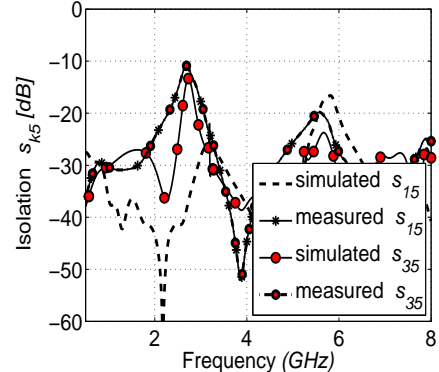

(b)
Figure 3: (a) Simulated and measured reflection coefficient of UWB sensing antenna(b) Mutual coupling between sensing and MIMO antennas.

The sensing and the communication antennas are working sequentially in CR applications. With different capacitance values, slight variation in the resonance curves of the UWB antenna was observed. As the UWB sensing antenna covered much wider band than the required band of interest hence this variation was insignificant for the overall $\mathrm{CR}$ operation.

For the sensing mode, it is necessary to have an omni-directional radiation pattern to increase the coverage. Figs. 4 (a), (b), (c) and (d) show the simulated 3-D gain patterns of the UWB antenna at four different frequency bands: $1.5 \mathrm{GHz}, 2.0 \mathrm{GHz}, 3.0 \mathrm{GHz}$ and 4.0 $\mathrm{GHz}$, respectively. All the MIMO antenna elements were terminated with $50 \Omega$ loads when we were measuring gain patterns and radiaiton efficiencies of the sensing antenna. It can be seen from the simulated results that the sensing antenna is exhibiting omni-directional pattern at four different frequencies. Simulated and measured 2-D gain patterns in the $y z$-plane at two different frequencies $(1.5 \mathrm{GHz}, 3.0$ $\mathrm{GHz}$ ) are shown in Fig. 5. Simulated and measured peak gains and radiation efficiencies $(\% \eta)$ for the sensing antenna are computed and summarized in Table I. Very good agreement is observed.

For the sensing antenna, we have shown the simulated and measured gain and efficiencies at four frequencies at $1.5 \mathrm{GHz}, 2 \mathrm{GHz}$, $3 \mathrm{GHz}$ and $4 \mathrm{GHz}$ to get the overall picture of the antenna's perforamnce. However, we have also computed the simulated radiaiton efficiency at the boundary of $-6 d B$ matching to check the worst case efficiency. The simulated efficiencies computed at the $-6 d B$ edges of $0.75 \mathrm{GHz}$ and $7.65 \mathrm{GHz}$ were $72 \%$ and $93.26 \%$, respectively. This showed the good radiation characteristics of the UWB sesning antenna even at the band edges.

Table I: Antenna efficiency and peak gain of the sensing antenna

\begin{tabular}{|c|c|c|c|c|}
\hline \multirow{2}{*}{} & \multicolumn{2}{|c|}{ Peak Gain $(\mathrm{dBi})$} & \multicolumn{2}{c|}{ Efficiency $(\% \eta)$} \\
\cline { 2 - 5 } & Simulated & Measured & Simulated & Measured \\
\hline $1.5 \mathrm{GHz}$ & 2.36 & 2.1 & 85 & 78 \\
\hline $2.0 \mathrm{GHz}$ & 3.55 & 3.28 & 91 & 84 \\
\hline $3.0 \mathrm{GHz}$ & 4.51 & 4.01 & 93 & 84 \\
\hline $4.0 \mathrm{GHz}$ & 5.29 & 4.89 & 95 & 87 \\
\hline
\end{tabular}

\section{B. MIMO Antenna Scattering Parameters}

The simulated reflection coefficient curves for Ant-1 are shown in Fig. 6(a). Since all of the 4-element MIMO antennas are identical and placed symmetrically on the substrate, almost same results were obtained from all ports. Varactor diodes were used to reactively 


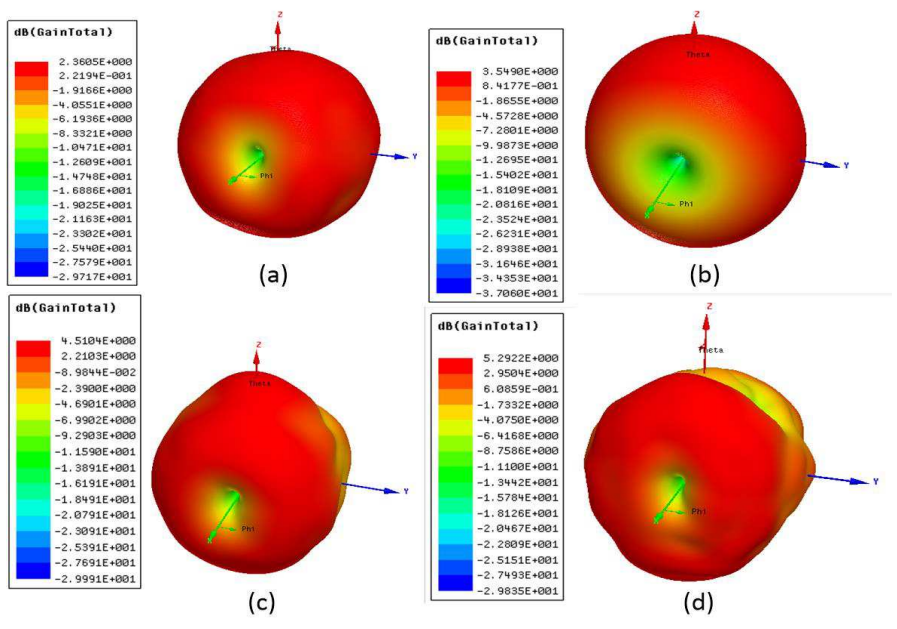

Figure 4: Simulated 3-D gain pattern at (a) $1.5 \mathrm{GHz}$ (b) $2.0 \mathrm{GHz}$ (c) $3.0 \mathrm{MHz}$ (d) $4.0 \mathrm{GHz}$.

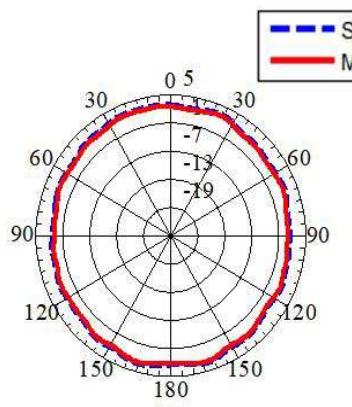

(a)
Simulated

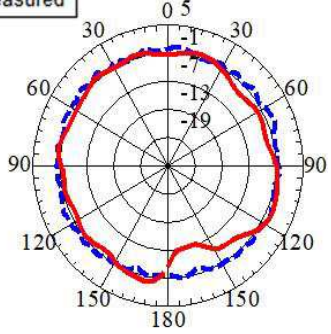

(b)
Figure 5: Simulated and measured 2D gain pattern in $y z$-plane (a) $1.5 \mathrm{GHz}$ (b) $3.0 \mathrm{GHz}$.

load the slots by varying the capacitance value via the reverse bias voltage. The MIMO reconfigurable antenna resonating frequencies were varied over a wide frequency band from $1.77 \mathrm{GHz}$ to $2.51 \mathrm{GHz}$ with minimum $-6 \mathrm{~dB}$ operating $\mathrm{BW}$ of $45 \mathrm{MHz}$. The voltage values used for varactor diode biasing for antenna tuning were $0 \mathrm{~V}, 1 \mathrm{~V}, 3 \mathrm{~V}$, $4 \mathrm{~V}, 6 \mathrm{~V}$ and $10 \mathrm{~V}$ with the corresponding varactor diode capacitance values of $0.9 p F, 1.1 p F, 1.45 p F, 1.82 p F, 3.28 p F$ and $5.08 p F$.

The measured reflection coefficient curves for the MIMO antenna system are shown in Fig. 6(b). The measured frequency bands obtained were from 1.76 2.52 $\mathrm{GHz}$ with minimum $-6 \mathrm{~dB}$ operating BW of $50 \mathrm{MHz}$. The simulated and measured $S_{33}$ and $S_{44}$ are almost similar to $S_{11}$. The maximum shift observed between simulated and measured results was about $35 \mathrm{MHz}$ over the entire band. As the resonace frequencies are smoothly varied over entire band from 1.77 $\mathrm{GHz}$ to $2.51 \mathrm{GHz}$, any shift observed can be easily compensated for as we have a continuous sweep of antennas resonances.

The proposed MIMO antenna system was also evaluated for mutual coupling among various antenna elements. The simulated and measured isolation curves for closely spaced antenna elements, Ant1 and Ant-2, are shown in Figs. 7(a) and 7(b), respectively. The coupling value at $1.8 \mathrm{GHz}$ was $-9.5 \mathrm{~dB}$ while it improved to -13 $d B$ at $2.45 \mathrm{GHz}$ band. Mutual coupling values of more than $10 \mathrm{~dB}$ were observed between other antenna elements like Ant-1 \& Ant-3 and Ant- 1 \& Ant- 4 in all the operating bands. As all the antenna elements are similar in structure and placed symmetrically, the $S_{34}$ curves obtained were similar to $S_{12}$.
The antenna elements should be as isolated as possible. But due to the area constraint, we need to get isolation that is sufficient to ensure good MIMO performance. The differences between the simulated and measured $S_{12}$ results are probably due to the differences in substrate properties of the fabricated and modeled antennas as well as fabrication tolerances. The connectors were hand soldered and placed. In addition, differences might be because of the lack of modeling flexibility of the varactor diodes in HFSS. The varactor diode was modeled as a series resistor with a variable capacitor. The series resistance value used was from 0.4 to 1.2 while the capacitance values used were from $0.9 p F$ to $5.08 p F$ for different biasing conditions. The modeling does not take into account the losses and packaging effects that might lead to slight differences between simulated and measured results.

The integration of 2-element MIMO antennas (Ant-1 \& Ant-2) within the sensing antenna was crucial to analyze in order understand the slot effects on the UWB antenna performance. The analysis was carried out using surface current distribution without (Case-1) and with (Case-2) slots on the sensing antenna at $2.45 \mathrm{GHz}$. Figs. 8(a) and 8 (c) show the current distribution on the surface of the UWB antenna while Figs. 8(b) and 8(d) show the current distribution on the GND plane of the UWB antenna for the given two cases. It is clear from the figures that most of the current distribution is around the sensing antenna lower edges and the coupling between sensing antenna with other ports was insignificant. The edges were mainly contributing towards the radiation. Hence, the slots are not affecting the radiating behavior of the UWB sensing antenna.

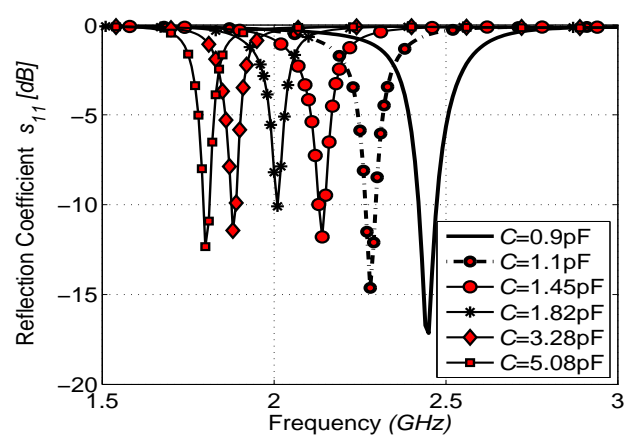

(a)

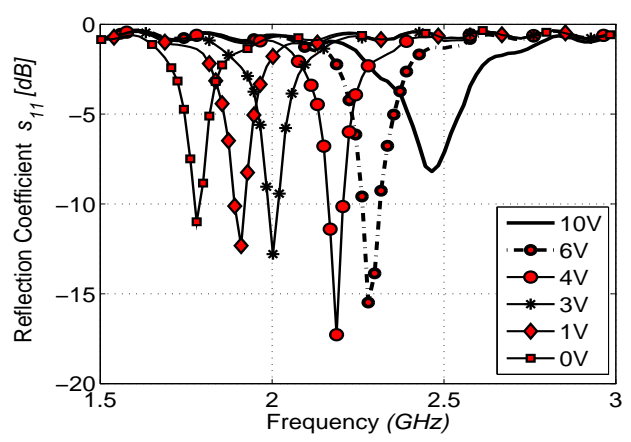

(b)

Figure 6: (a) $\left|s_{11}\right|$ - simulated (b) $\left|s_{11}\right|$ - measured

\section{Far Field Radiation Characteristics of the MIMO Antenna}

For the proposed reconfigurable MIMO antenna, 3-D gain patterns were computed using HFSS. The gain patterns and efficiency values were also measured for the proposed MIMO antenna system using a SATIMO Starlab anechoic chamber. A comparison between simulated and measured peak gains and efficiencies are provided in Table II. A good agreement between simulated and measured values is observed. 


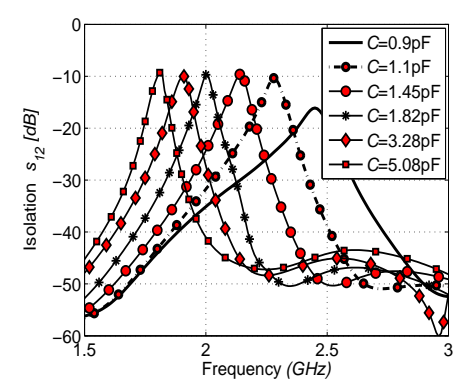

(a)

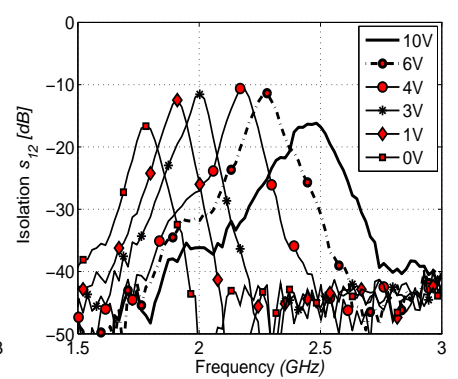

(b)
Figure 7: a) $\left|s_{12}\right|$-simulated (b) $\left|s_{12}\right|$ - measured

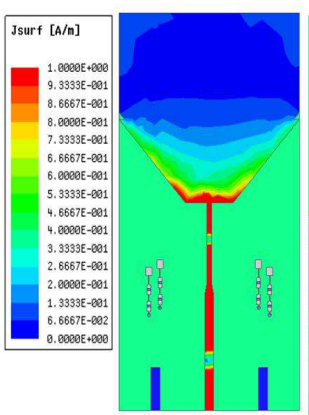

(a)

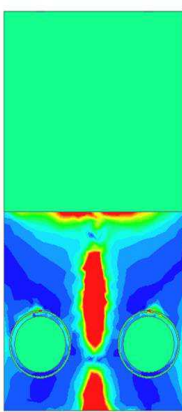

(b)

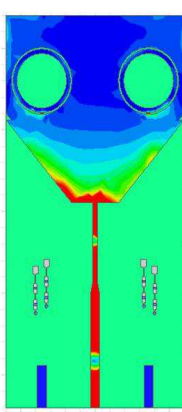

(c)

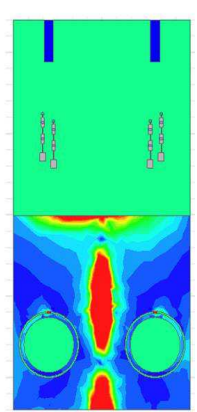

(d)
Figure 8: Surface current distribution at $2.45 \mathrm{GHz}$ (a) Sensing Antenna without slots (b) GND plane wihtout sensing slots (c) Sensing Antenna with slots (d) GND plane wiht sensing slots.

The simulated and measurement frequencies are designated as $\left(f_{i s}\right)$ and $\left(f_{i m}\right)$, respectively, where $\mathrm{i}=1,2 \ldots .5$. The $f_{i s}$ values used were $\left(f_{1 s}=1.8 \mathrm{GHz}, f_{2 s}=1.88 \mathrm{GHz}, f_{3 s}=2.01 \mathrm{GHz}, f_{4 s}=2.14 \mathrm{GHz}, f_{5 s}=2.46\right.$ $\mathrm{GHz})$ and $f_{i m}$ used were $\left(f_{1 m}=1.78 \mathrm{GHz}, f_{2 m}=1.910 \mathrm{GHz}, f_{3 m}=2.002\right.$ $\mathrm{GHz}, f_{4 m}=2.188 \mathrm{GHz}, f_{5 m}=2.45 \mathrm{GHz}$ ), respectively. The gain pattern measurement setup is shown in Fig. 9.

For the MIMO antenna system, we have measured the peak gains and radiation efficiencies at five resonance frequencies to shows the antenna behavior over the entire band. In addition, we have also computed the boundary frequency for $-6 d B$ matching for 2 resonance bands to get an idea of the worst case antenna behavior. For the 2 bands with center frequency at $1.8 \mathrm{GHz}$ and $2.44 \mathrm{GHz}$, the boundary of $-6 \mathrm{~dB}$ matching efficiencies were $(1.77 \mathrm{GHz}$ and 1.82 $\mathrm{GHz}$ ) and (2.39 $\mathrm{GHz}$ and $2.51 \mathrm{GHz})$, respectively. The efficiency values computed at $1.77 \mathrm{GHz}$ and $1.82 \mathrm{GHz}$ were $73 \%$ and $75 \%$, respectively, while $83 \%$ and $84.5 \%$ efficiency values were found at $2.39 \mathrm{GHz}$ and $2.51 \mathrm{GHz}$, respectively. The simulated efficiency values computed for the given MIMO antenna indicate the efficient radiation from the antenna elements over the entire $-6 d B$ operating bandwidth. The differences between simulated and measured peak gain and efficiency values in Table II were mainly because of the computation of these values in an ideal HFSS simulation environment compared to the practical anechoic chamber scenario. The effects of mast wires and other connectors in measurement setup near the antenna assembly were not considered in HFSS. In addition, the aforementioned differences in substrate properties of the fabricated antenna and fabrication tolerances might have contributed to this deviation.

The simulated and measured normalized 2D gain patterns, for frequency band at $1.78 \mathrm{GHz}$ are given in Fig. 10. The patterns show the $x-y$ and $y-z$ planes for 4-elements (Ant-1, Ant-2, Ant-3 and Ant4). The simulated and measured $\theta$-cut curves for $\phi=0^{\circ}$, at $1.78 \mathrm{GHz}$.

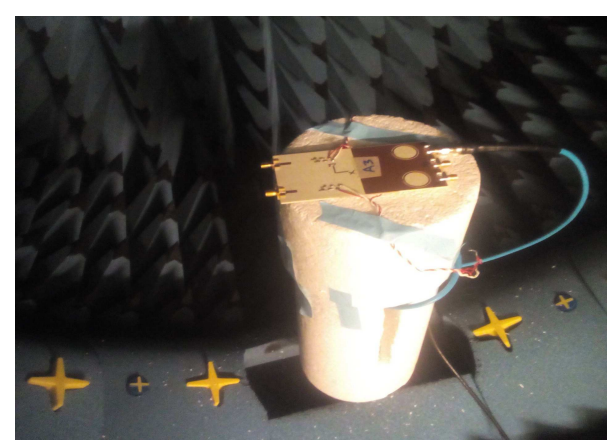

Figure 9: Radiation pattern measurement setup.

Table II: Efficiency and peak gain of the MIMO antenna system

\begin{tabular}{|l|c|c|c|c|}
\hline & \multicolumn{2}{|c|}{ Peak Gain (dBi) } & \multicolumn{2}{c|}{ Efficiency (\%) } \\
\cline { 2 - 5 } & Simulated & Measured & Simulated & Measured \\
\hline$f_{1 s}, f_{1 m}$ & 1.5 & 0.5 & 75 & 65 \\
\hline$f_{2 s}, f_{2 m}$ & 1.85 & 1.45 & 78 & 68 \\
\hline$f_{3 s}, f_{3 m}$ & 2.1 & 1.78 & 80 & 71 \\
\hline$f_{4 s}, f_{4 m}$ & 3.29 & 3.1 & 83 & 79 \\
\hline$f_{5 s}, f_{5 m}$ & 3.89 & 3.2 & 87 & 81 \\
\hline
\end{tabular}

are shown in Figs. 10(a) and 10(c), while $\phi$-cut curves for $\theta=90^{\circ}$, at $1.78 \mathrm{GHz}$ are shown in Figs. 10(b) and 10(d), respectively. A tilt in the patterns is visible which is usually desirable for good MIMO performance.

\section{ECC and TARC for MIMO Antenna}

The correlation coefficient is a measure of how the MIMO channels are correlated. The envelop correlation coefficient (ECC) can be calculated using the radiation patterns of the antennas as given in [15]. For the given MIMO antenna system, ECC values were calculated using the simulated and measured radiation patterns. The ECC values for different bands are given in Table III. Good MIMO performance is observed as the ECC values did not exceed 0.248 across the complete operating band.

Another important parameter for MIMO operation characterization is the total active reflection coefficient (TARC) [15] and is given by:

$$
\Gamma_{a}^{t}=\frac{\sqrt{\sum_{i=1}^{N}\left|b_{i}\right|^{2}}}{\sqrt{\sum_{i=1}^{N}\left|a_{i}\right|^{2}}}
$$

where $a_{i}$ and $b_{i}$ are the incident signals and reflected signals, respectively.

TARC curves were obtained by exciting one port at $1 \mathrm{e}^{j 0}$ while the phases of the other ports were changed to different phase excitations. The simulated and measured TARC curves are shown in Figs. 11(a) and 11(b), respectively. The excitation phases used were chosen from $0^{\circ}, 30^{\circ}, 60^{\circ}, 90^{\circ}, 150^{\circ}, 180^{\circ}, 240^{\circ}$ and $300^{\circ}$ for all four ports $(P-1$, $P-2, P-3, P-4)$ to form 6-possible cases. The TARC curves describe the effective BW of the MIMO antenna system. It is clear that the operating BW of the proposed MIMO antenna system is robust and not severely affected by phase changes in the excitation of the other ports. The cases considered were:

Case- $1: P-1=0^{\circ} ; P-2=0^{\circ} ; P-3=30^{\circ} ; P-4=60^{\circ}$

Case $-2: P-1=0^{\circ} ; P-2=30^{\circ} ; P-3=90^{\circ} ; P-4=150^{\circ}$ 


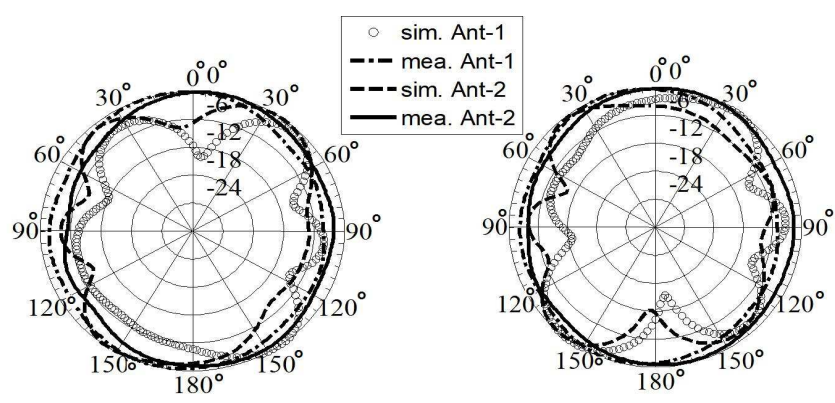

(a)

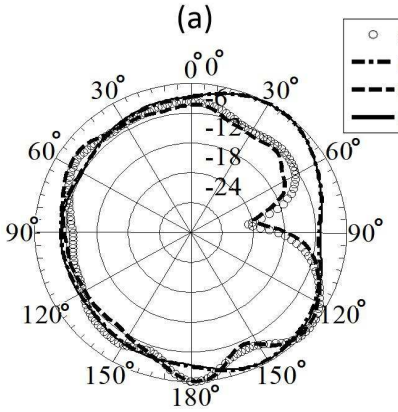

(c) (b)

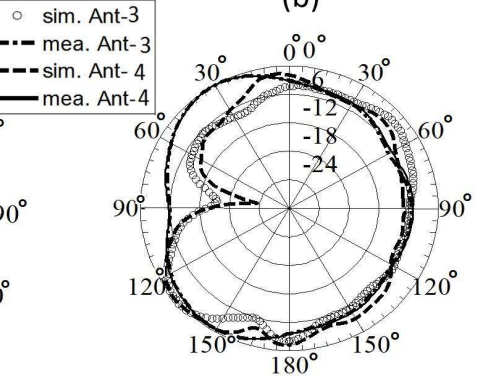

(d)
Figure 10: Simulated and measured normalized radiation patterns at $1.78 \mathrm{GHz}$ (Etotal) (a) ( $\theta$-cuts) Ant-1 and Ant-2 at $\phi=0^{\circ}$ (b) ( $\theta$-cuts) Ant-3 and Ant-4 at $\phi=0^{\circ}$ (c) ( $\phi$-cuts) Ant-1 and Ant-2 at $\theta=90^{\circ}$ (d) ( $\phi$-cuts) Ant-3 and Ant- 4 at $\theta=90^{\circ}$

Table III: Simulated and measured ECC

\begin{tabular}{|l|l|l|l|l|l|l|l|}
\hline \multicolumn{4}{|c|}{ Simulated ECC } & \multicolumn{4}{c|}{ Measured ECC } \\
\hline freq. & $\mathrm{ECC}_{12}$ & $\mathrm{ECC}_{13}$ & $\mathrm{ECC}_{14}$ & freq. & $\mathrm{ECC}_{12}$ & $\mathrm{ECC}_{13}$ & $\mathrm{ECC}_{14}$ \\
\hline$f_{1 s}$ & 0.009 & 0.093 & 0.025 & $f_{1 m}$ & 0.079 & 0.053 & 0.045 \\
\hline$f_{2 s}$ & 0.035 & 0.248 & 0.035 & $f_{2 m}$ & 0.061 & 0.131 & 0.014 \\
\hline$f_{3 s}$ & 0.062 & 0.186 & 0.075 & $f_{3 m}$ & 0.013 & 0.123 & 0.022 \\
\hline$f_{4 s}$ & 0.11 & 0.16 & 0.036 & $f_{4 m}$ & 0.045 & 0.114 & 0.098 \\
\hline$f_{5 s}$ & 0.095 & 0.171 & 0.091 & $f_{5 m}$ & 0.082 & 0.081 & 0.038 \\
\hline
\end{tabular}

Case- $3: P-1=0^{\circ} ; P-2=60^{\circ} ; P-3=150^{\circ} ; P-4=180^{\circ}$

Case-4: $P-1=0^{\circ} ; P-2=60^{\circ} ; P-3=90^{\circ} ; P-4=180^{\circ}$

Case-5: $P-1=0^{\circ} ; P-2=90^{\circ} ; P-3=150^{\circ} ; P-4=240^{\circ}$

Case-6: $P-1=0^{\circ} ; P-2=30^{\circ} ; P-3=270^{\circ} ; P-4=300^{\circ}$

These cases were taken for the $0.9 \mathrm{pF}$ varactor value in simulation compared to $10 \mathrm{~V}$ bias case in measurement. Other combination/values showed similar BW stability.

\section{Conclusions}

In this paper, an integrated CR antenna system is presented. The proposed design consisted of an UWB modified monopole based sensing antenna and frequency agile slot-based 4-element MIMO communication antenna system that are integrated within each other. The complete antenna system is compact, low profile, planar in structure and fabricated on a single substrate board of dimensions $60 \times 120 \times 1.5 \mathrm{~mm}^{3}$. The reconfigurable MIMO antenna exhibited a wide tuning capability over a frequency band of $1.77 \mathrm{GHz}$ to 2.51 $\mathrm{GHz}$ covering several well-known frequency bands while sensing antenna covered frequency band from $0.75 \mathrm{GHz}$ to $7.65 \mathrm{GHz}$. Good MIMO performance metrics was observed.

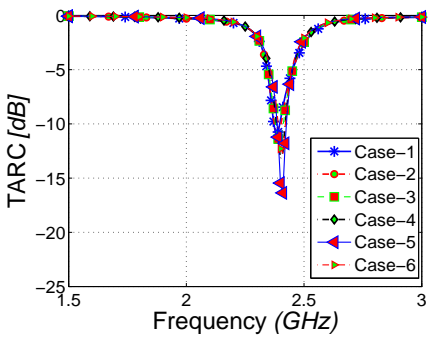

(a)

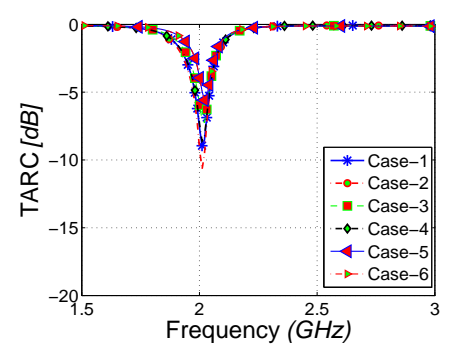

(b)
Figure 11: TARC curves for the proposed MIMO antenna system. (a) Simulated at $0.9 p F$ (b) Measured at $10 \mathrm{~V}$.

\section{ACKNOWLEDGMENT}

This work is supported in part by the Deanship of Scientific Research (DSR) at King Fahd University of Petroleum and Minerals (KFUPM) under project number KAUST002 and in part by the King Abdullah University of Science and Technology (KAUST) Office of Sponsored Research (OSR) under Award No. OSR 2016-KKI-2899.

\section{REFERENCES}

[1] J. Mitola, "Cognitive radio architecture evolution," Proc. of the IEEE, vol. 97, no. 4, pp. 626-641, 2009.

[2] P. Steenkiste, D. Sicker, G. Minden, and D. Raychaudhuri, "Future directions in cognitive radio network research," NSF Workshop Report, vol. 4, no. 1, pp. 1-2, 2009.

[3] J.-H. Lim, Z.-J. Jin, C.-W. Song, and T.-Y. Yun, "Simultaneous frequency and isolation reconfigurable mimo pifa using pin diodes," IEEE Trans. Antennas Propag., vol. 60, no. 12, pp. 5939-5946, 2012.

[4] H. T. Chattha, M. Nasir, Q. H. Abbasi, Y. Huang, and S. S. Alja'afreh, "Compact low profile dual port single wideband planar inverted-f mimo antenna," IEEE Antennas Wireless Propag. Lett., vol. 12, pp. 1673-1675, 2005.

[5] S. Soltani, P. Lotfi, and R. D. Murch, "A port and frequency reconfigurable mimo slot antenna for wlan applications," IEEE Trans. Antennas Propag., vol. 64, no. 4, pp. 1209-1217, 2016.

[6] A. N. Kulkarni and S. K. Sharma, "Frequency reconfigurable microstrip loop antenna covering lte bands with mimo implementation and wideband microstrip slot antenna all for portable wireless dtv media player," IEEE Trans. Antennas Propag., vol. 61, no. 2, pp. 964-968, 2013.

[7] Y. Tawk, J. Costantine, K. Avery, and C. Christodoulou, "Implementation of a cognitive radio front-end using rotatable controlled reconfigurable antennas," IEEE Trans. Antennas Propag., vol. 59, no. 5, pp. 1773-1778, 2011.

[8] R. Hussain and M. S. Sharawi, "Integrated reconfigurable multipleinput-multiple-output antenna system with an ultra-wideband sensing antenna for cognitive radio platforms," IET Microw. Antennas Propag., vol. 9, no. 9, pp. 940-947, 2015.

[9] Y. Tawk, J. Costantine, and C. Christodoulou, "Reconfigurable filtennas and mimo in cognitive radio applications," IEEE Trans. Antennas Propag., vol. 62, no. 3, pp. 1074-1084, 2014.

[10] R. Hussain and M. S. Sharawi, "A cognitive radio reconfigurable mimo and sensing antenna system," IEEE Antennas Wireless Propag. Lett., vol. 14 , pp. $257-260,2015$.

[11] _ _ "Reconfigurable mimo antennas for cognitive radios," Spectrum Access and Management for Cognitive Radio Networks, pp. 233-264, 2017.

[12] J.-M. Lee, K.-B. Kim, H.-K. Ryu, and J.-M. Woo, "A compact ultrawideband mimo antenna with wlan band-rejected operation for mobile devices," IEEE Antennas Wireless Propag. Lett., vol. 11, pp. 990-993, 2012.

[13] C.-T. Chuang, T.-J. Lin, and S.-J. Chung, "A band-notched uwb monopole antenna with high notch-band-edge selectivity," IEEE Trans. Antennas Propag., vol. 60, no. 10, pp. 4492-4499, 2012.

[14] K. S. Ryu and A. A. Kishk, "Uwb antenna with single or dual bandnotches for lower wlan band and upper wlan band," IEEE Trans. Antennas Propag., vol. 57, no. 12, pp. 3942-3950, 2009.

[15] M. S. Sharawi, "Printed mimo antenna engineering," Artech House, 2014. 